Manuelle Medizin 2020 · 58:251

https://doi.org/10.1007/s00337-020-00720-3

Online publiziert: 27. Juli 2020

(c) Springer Medizin Verlag GmbH, ein Teil von Springer Nature 2020

\section{Erwiderung}

Zum Leserbrief von Sacher et al (2020) Zeitschrift Manuelle Medizin - quo vadis? https:// doi.org/10.1007/s00337-020-00719-w

\section{Originalbeitrag}

Themenheft "Manuelle Medizin bei Kindern", Ausgabe $3 / 2020$

Sehr geehrter, lieber Herr Sacher, sehr geehrte Kollegen als Mitautoren im Diskussionsbeitrag zu Heft 3/2020 (Themenheft „Manuelle Medizin bei Kindern“),

herzlichen Dank für Ihren offenen und konstruktiven Diskussionsbeitrag.

Insbesondere haben wir uns über Ihre klare Aussprache für eine zukünftige aktive Mitarbeit bei der weiteren Entwicklung unserer Zeitschrift gefreut. Ähnliche Zuschriften sind bisher eher selten gewesen.

Offensichtlich ist die Neuausrichtung der Zeitschrift hin zu einem einerseits stärker international orientierten $\mathrm{Pu}$ blikationsorgan und andererseits im Rahmen von Prävention sowie funktionsorientierter Diagnostik und Therapie am Bewegungssystem breiter aufgestellten Journal eine objektive Notwendigkeit. Daher ist es auch offenbar kein Zufall, dass die von Ihnen angesprochenen Punkte Gegenstand aktueller Diskussionen sind. Unser Ziel ist es, gemeinsam mit dem Verlag ein tragfähiges und zukunftweisendes Konzept für die Zeitschrift zu entwickeln.

Die von Ihnen vorgetragenen Argumente und Vorschläge entsprechen also genau diesem Vorhaben.

\author{
L. Beyer' $\cdot$ A. Sammer ${ }^{2}$ \\ ${ }^{\top}$ Ärztehaus Mitte, Jena, Deutschland \\ ${ }^{2}$ Lehrstuhl für makroskopische und klinische Anatomie, Medizinische Universität, Graz, Österreich
}

\title{
Die Zukunft von Manuelle Medizin
}

- In das zu entwickelnde Konzept fließen folgende Überlegungen ein: Die Zeitschrift Manuelle Medizin soll sich hin zu einem „European Journal of Manual Medicine" entwickeln. Damit soll die Manuelle Medizin nicht nur die deutschsprachigen Gesellschaften repräsentieren, sondern auch die vorhandene Potenz der europäischen Strukturen der European Scientific Society of Manual Medicine (ESSOMM), des Multidisciplinary Joint Committee (MJC) Manual Medicine bei der European Union of Medical Specialists (UEMS) und der internationalen Akademie für Muskuloskeletale Manuelle Medizin (IAMMM) integrieren.

- Wir hoffen, so die Voraussetzung für eine Aufnahme in die Reihe gelisteter Zeitschriften zu schaffen. Dafür benötigen wir qualitativ hochwertige Publikationen in englischer Sprache, am besten als Open-Access-Publikation. Hierfür haben sich die allgemeinen Voraussetzungen verbessert, da die Kosten für OpenAccess-Veröffentlichungen über das Projekt DEAL [1] nicht mehr von den Autoren selbst getragen werden müssen. Zudem gibt es verschiedene Vereinbarungen mit Wissenschaftsstandorten wie Österreich, United Kingdom, Polen, Ungarn u. w.

Denkbar ist auch eine strikte Teilung der Zeitschrift in

- einen wissenschaftlichen, englischsprachigen, primär Open-Access-Teil mit ausführlichen Originalien und Übersichten sowie

- einen praxisorientierten Teil mit Kurzbeiträgen, Mitteilungen und Weiterbildungsartikeln.
Eine stärkere Onlinebegleitung der Zeitschrift durch z.B. Webinare, Behandlungsvideos oder ausführliche Bilderstrecken ist ebenfalls denkbar.

Alle diese Komponenten ließen sich kombinieren und ergäben zusammen eine zukunftsweisende, die Wissenschaft stärkende und zugleich mitgliederorientierte neue Manuelle Medizin.

Gemeinsam mit den Autoren des Zirkels für Manuelle Medizin und Entwicklungstherapie (ZIMMT-Gruppe) möchten wir alle Kolleginnen und Kollegen einladen, am Diskurs zur weiteren Entwicklung der Zeitschrift Manuelle Medizin teilzunehmen, ganz gleich, wo Sie Ihren persönlichen manualmedizinischen Schwerpunkt setzen. Jeder wird gebraucht!

L. Beyer

A. Sammer

\section{Korrespondenzadresse}

Prof. Dr. L. Beyer

Ärztehaus Mitte

Westbahnhofstr. 2, 07745 Jena, Deutschland lobeyer@t-online.de

\section{Dr. A. Sammer}

Lehrstuhl für makroskopische und klinische Anatomie, Medizinische Universität Harrachgasse 21/1 HG, 8010 Graz, Österreich andreas.sammer@medunigraz.at

Interessenkonflikt. L. Beyer und A. Sammer geben an, dass kein Interessenkonflikt besteht.

\section{Literatur}

1. https://www.projekt-deal.de/aktuelles/. Zugegriffen: 30 . Juni 2020 